\title{
ADJOINT-BASED DESIGN OF SHOCK MITIGATION DEVICES
}

\author{
Rainald Lohner ${ }^{\mathrm{a}}$ \\ ${ }^{a}$ CFD Center, Dept. of Computational and Data Science. College of Science, George Mason \\ University.Fairfax, VA, USA
}

\begin{abstract}
Unsteady primal and adjoint Euler solvers have been combined in order to aid in the design of shock mitigation devices.

The flowfield is integrated forward in time and stored. The adjoint is then integrated going backwards in time, restoring and interpolating the saved Euler solution to the current point in time.

The gradient is obtained from a surface integral formulation during the adjoint run. The technique has proven useful for the design of passive shock mitigation devices such as protective walls or curtains, as well as active shock mitigation devices, such as secondary explosions. Furthermore, algorithms have been developed to reduce the rather large storage demands inherent to the technique.

Several examples demonstrate the usefulness of the proposed approach.
\end{abstract}

